\title{
Rapid antimicrobial susceptibility testing of urinary tract isolates and samples by flow cytometry
}

\author{
CHRISTIAN GAUTHIER*, YVES ST-PIERRE and RICHARD VILLEMUR
}

INRS-Institut Armand-Frappier, Laval (Québec), Canada H7V 1B7

\begin{abstract}
A multiparametric flow cytometry antimicrobial susceptibility test was developed and its performance was evaluated on clinical urine isolates and samples in comparison with standard methods. Alterations in cytoplasmic membrane integrity were monitored by propidium iodide, and the anionic probe bis-(1,3-dibutylbarbituric acid) trimethine oxonol $\left(\mathrm{DiBAC}_{4}(3)\right)$ was used to measure changes in membrane potential. Microbial size and cellular content were analysed by light scattering. Twelve antibiotics were tested on 6 ATCC control strains, 22 urine isolates and 19 clinical urine samples, variously containing Escherichia coli, Pseudomonas aeruginosa, Klebsiella pneumoniae, Proteus mirabilis, Enterococcus faecalis, Staphylococcus aureus, S. saprophyticus and $S$. epidermidis. Agreement between the flow cytometry results, broth microdilution and disk diffusion tests was $93.9 \%(n=328$ tests $)$. Of the 20 discrepancies observed, 18 were for species other than $E$. coli. Perfect correlation was obtained with five antibiotics, whereas norfloxacin, nitrofurantoin and tetracycline were responsible for $13(65 \%)$ of the 20 discrepancies.
\end{abstract}

\section{Introduction}

Antimicrobial susceptibility testing (AST) of bacteria isolated from urinary tract infection (UTI) samples is usually done by standardised disk diffusion or broth dilution. An alternative strategy is to detect directly the effects of an antibiotic on bacterial cells by using specific fluorescent molecules [1]. Propidium iodide (PI) can enter microbial cells that have lost their membrane integrity following antimicrobial exposure, and can bind to nucleic acids [2,3]. Bis-(1,3-dibutylbarbituric acid) trimethine oxonol $\left(\mathrm{DiBAC}_{4}(3)\right)$ is a negatively charged hydrophobic molecule that binds to membranes and the intracellular proteins of depolarised cells, resulting in increased fluorescence emission $[4,5]$. The fluorescent cells can then be detected by epifluorescence microscopy or laser flow cytometry. The advantages of flow cytometry (FC) are that it can detect and enumerate thousands of cells in a few seconds, and can monitor up to four fluorescent probes at the same time. Light scattering and fluorescence

Received 7 February 2001; revised version accepted 25 July 2001.

Corresponding author: Dr R. Villemur (e-mail: richard. villemur@inrs-iaf.uquebec.ca).

* Present address: Institut de Recherches Cliniques de Montréal, 110 avenue des Pins ouest, Montréal (Québec), Canada H2W $1 \mathrm{R} 7$. measurements are indicative both of the morphological and physiological states of the individual cells, and are acquired and analysed in real time by a computer system $[6,7]$.

This study aimed to use FC to reduce the analysis time of ASTs. A flow cytometric susceptibility testing (FCAST) method was established by monitoring the antimicrobial effects on bacterial cells with two fluorescent dyes, PI and $\mathrm{DiBAC}_{4}(3)$. This method was applied to urine isolates and urine samples, and the results were compared with those obtained by two conventional AST methods.

\section{Materials and methods}

\section{Bacterial strains and media}

Control strains were from the American Type Culture Collection (ATCC). Bacterial isolates from urine, and infected urine samples (Table 2) were collected from UTIs and were provided by the Department of Infectious Diseases and Microbiology at the Hôpital Maisonneuve-Rosemont (University of Montreal, Montreal, QC, Canada). Infected urine samples contained $>10^{5} \mathrm{cfu} / \mathrm{ml}$ and were kept at $4{ }^{\circ} \mathrm{C}$ in sterile tubes for no longer than $24 \mathrm{~h}$ before assays. Bacteria were cultured in Mueller-Hinton Broth (BBL, Franklin Lakes, NJ, USA) supplemented with cations (MHSC: 
$\mathrm{CaCl}_{2} 50 \mathrm{mg} / \mathrm{L}, \mathrm{MgCl}_{2} 20 \mathrm{mg} / \mathrm{L}$ ). Bacterial viability was assessed by performing spread-plate counts on Trypticase Soy Agar (TSA); Difco Laboratories, Detroit, MI, USA.

\section{Antibiotics and fluorescent dyes}

Ampicillin, gentamicin and tetracycline were from ICN (Mississauga, ON, Canada), carbenicillin from Pfizer (Ste-Anne-de-Bellevue, QC, Canada), ceftazidime from Lilly (Scarborough, ON, Canada), ceftriaxone from Hoffman-La Roche (Mississauga, ON, Canada), nitrofurantoin, norfloxacin, oxacillin, penicillin G, trimethoprim, sulphamethoxazole and vancomycin from Sigma. Antibiotic stock solutions $(5 \mathrm{mg} / \mathrm{ml})$ were made with the appropriate diluents, as listed by the NCCLS [8] and kept at $-20^{\circ} \mathrm{C}$. $\operatorname{DiBAC}_{4}(3)$ (Molecular Probes, Eugene, OR, USA) was dissolved initially in dimethyl sulphoxide and diluted in ethanol to $1 \mathrm{mg} / \mathrm{ml}$. The PI (Sigma) stock solution $(1 \mathrm{mg} / \mathrm{ml})$ was made with Milli-Q water. Solutions of fluorescent probes were kept at $4^{\circ} \mathrm{C}$ and were stable for up to 6 months when protected from light.

\section{Reference susceptibility tests}

Disk susceptibility tests were performed at the Department of Infectious Diseases and Microbiology (Hôpital Maisonneuve-Rosemont) by the NCCLS protocol [8]. Broth microdilution susceptibility tests were done to determine the minimum inhibitory concentration (MIC), again according to the NCCLS guidelines [8].

\section{$F C-A S T$}

Three-to-five colonies from TSA, or $100 \mu \mathrm{l}$ of infected urine, were added to $5 \mathrm{ml}$ of MHSC broth and incubated for $c .2 \mathrm{~h}$ with shaking at $37^{\circ} \mathrm{C}$ and $250 \mathrm{rpm}$. These cultures were then adjusted to the turbidity of a 0.5 McFarland standard and diluted five-fold in MHSC broth; $500 \mu \mathrm{l}$ of this dilution was then mixed with $500 \mu \mathrm{l}$ of MHSC containing the appropriate concentration of antibiotic. The inoculum size was c. $5 \times$ $10^{6} \mathrm{cfu} / \mathrm{ml}$. The final antibiotic concentrations in the samples were equivalent to the NCCLS MIC breakpoints for susceptible interpretation (Table 2). The cultures were incubated for $2 \mathrm{~h}$ at $37^{\circ} \mathrm{C}$ and $250 \mathrm{rpm}$, centrifuged at $10000 \mathrm{~g}$ for $2 \mathrm{~min}$, then resuspended in $1 \mathrm{ml}$ of phosphate-buffered saline (PBS, $130 \mathrm{mM}$ sodium chloride, $10 \mathrm{mM}$ sodium phosphate buffer) $\mathrm{pH}$ 7.2 containing both $\operatorname{DiBAC}_{4}(3)(10 \mu \mathrm{g} / \mathrm{ml})$ and PI $(10 \mu \mathrm{g} / \mathrm{ml})$. Before use this diluent was filter-sterilised through a $0.22-\mu \mathrm{m}$ pore membrane. After incubation for $10 \mathrm{~min}$ in the dark at room temperature, the cultures were diluted in PBS to yield c. $10^{6}$ cells $/ \mathrm{ml}$. The controls were: (i) untreated and unstained bacterial cells for autofluorescence, (ii) untreated and stained bacterial cells (live control) and (iii) bacterial cells treated for $5 \mathrm{~min}$ with ethanol $70 \%$ (dead control) and stained with both dyes.

\section{Flow cytometry}

The Coulter Epics XL-MCL (Beckman Coulter, Fullerton, CA, USA) was used. The $\operatorname{DiBAC}_{4}(3)$ fluorescent signal was collected in the FL1 photomultiplier with a $525-\mathrm{nm}$ bandpass filter (adjusted at $720 \mathrm{~V}$ ) and the PI fluorescent signal was collected in the FL3 photomultiplier with a 620-nm bandpass filter (adjusted at $850 \mathrm{~V})$. Signal compensation that eliminates optical overlap between both dyes was $<10 \%$. The samples were run at a low pressure setting, corresponding to a flow rate of 100-200 events/s during the analysis; 10000 events were acquired for each analysis.

\section{Interpretative criteria for $F C$ - $A S T$}

Two acquisition regions were defined. The first was applied to the light-scattering profiles (region I; Fig. la, c, e, g), and was used to exclude events related to electronic noise and cell debris. The mean fluorescence intensity (MFI) of the PI and $\operatorname{DiBAC}_{4}(3)$ signals was measured only from events recorded within this region. The second acquisition region was defined on the biparametric fluorescence histograms (region II in Fig. $\mathrm{lb}, \mathrm{d}, \mathrm{f}, \mathrm{h})$. Events recorded in this region were fluorescent with both dyes and allowed the exclusion of non-fluorescent events (live cells) and events labelled with only one dye. Fluorescent events in acquisition region II represented cells that have lost both membrane integrity and polarisation.

The numbers of fluorescent events in region II and the MFI for the PI and $\operatorname{DiBAC}_{4}(3)$ signals were compared between the live controls and the antibiotic-treated samples. For each sample, a value $V_{i}$ was derived, defined by equation 1 :

$$
\mathrm{V}_{\mathrm{i}}=\mathrm{N}_{\mathrm{i}} \times \mathrm{MFI}_{\mathrm{PI}} \times \mathrm{MFI}_{\operatorname{DiBAC} 4(3)}
$$

where $\mathrm{N}_{\mathrm{i}}$ was the number of fluorescent events recorded in acquisition region $\mathrm{II}$, and $\mathrm{MFI}_{\mathrm{PI}}$ and $\mathrm{MFI}_{\text {DiBAC4(3) }}$ were the MFI values in acquisition region I for each dye. The comparative factor, $C_{i}$ was determined by equation 2 :

$$
\mathrm{C}_{\mathrm{i}}=\mathrm{V}_{\mathrm{i}} / \mathrm{V}_{\mathrm{L}}
$$

where $\mathrm{V}_{\mathrm{L}}$ was the $\mathrm{V}$ value determined by equation 1 for the live control sample.

A discrepancy was considered minor when: (1) intermediate resistance was indicated by the FC-AST and one of the standard antimicrobial tests (disk diffusion or microdilution) indicated resistance or susceptibility; (2) the FC-AST results indicated resistance and one of the standard tests indicated resistance and the other susceptibility; or (3) the FC-AST results indicated resistance or susceptibility and the standard tests indicated intermediate resistance. A discrepancy was considered major when: (1) susceptibility was indicated by FC-AST whereas one of the standard tests indicated susceptibility and the other resistance; or (2) FC-AST results indicated 

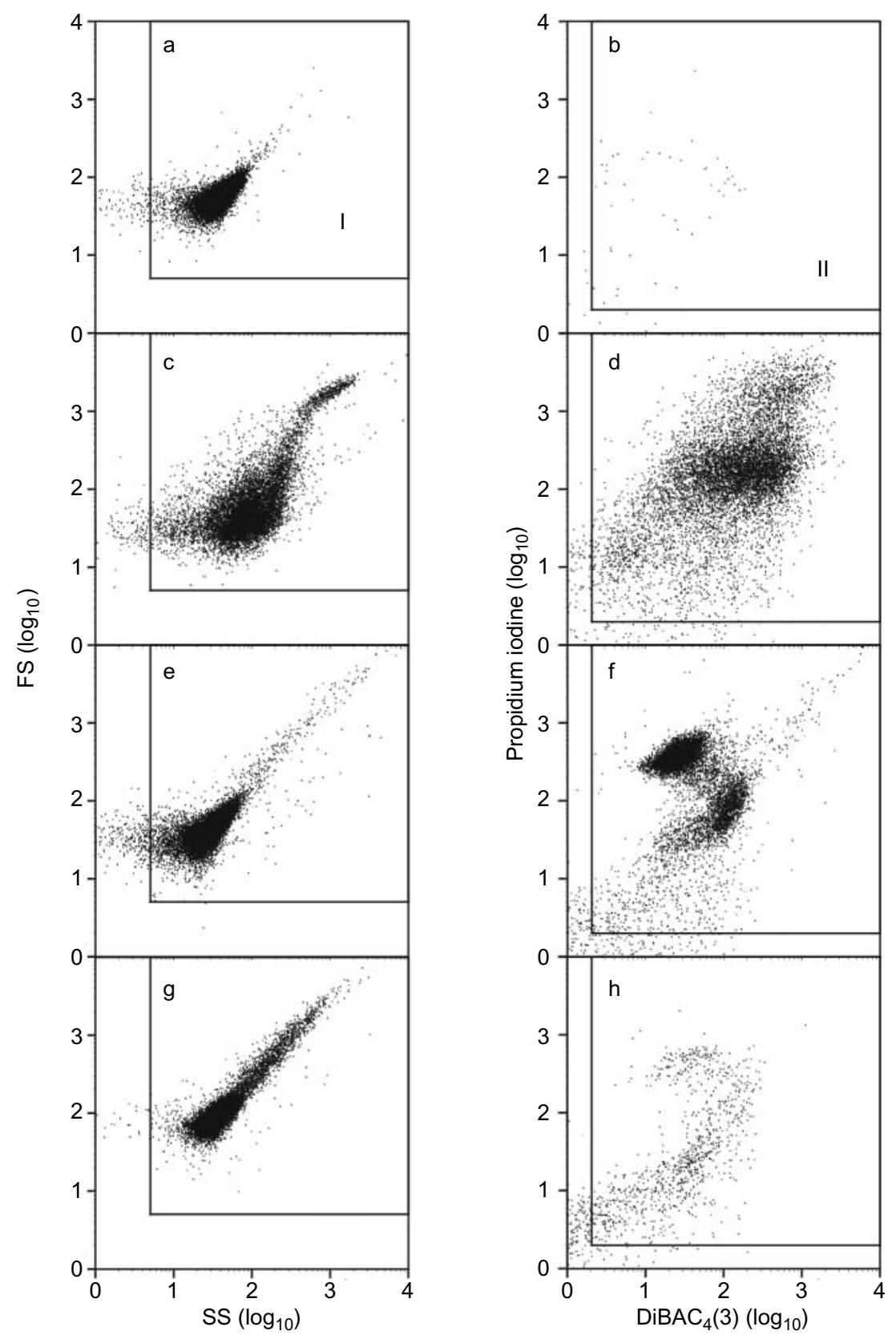

Fig. 1. Light-scattering profiles (forward scatter, FS, versus side scatter, SS) (a, c, e, g) and bi-parametric histograms (b, d, f, h) $\left(\operatorname{DiBAC}_{4}(3)\right.$ versus $\left.\mathrm{PI}\right)$ for $E$. coli 25922 untreated $(\mathbf{a}, \mathbf{b})$ or treated for $2 \mathrm{~h}$ with ampicillin (c, d), gentamicin (e, f) or cotrimoxazole $(\mathbf{g}, \mathbf{h})$, and analysed by FC. Events recorded in the acquisition region I in the light scattering-profiles were measured for fluorescence. Fluorescent events recorded in acquisition region II were taken as dead cells. The proportions of dead cells recorded in panels $\mathbf{b}, \mathbf{d}, \mathbf{f}$ and $\mathbf{h}$ were $0.5 \%, 89 \%, 79 \%$ and $9.6 \%$, respectively.

resistance whereas both standard tests indicated susceptibility. Finally, a discrepancy was considered very major when susceptibility was indicated by FC-AST and resistance by both the standard tests. The susceptibility interpretations for the two reference tests were based on the NCCLS performance standards (M100-S9) [8] and were controlled with the appropriate ATCC strains.

\section{Results}

\section{Interpretative criteria}

For each antibiotic, a specific range of the $C_{i}$ values (Table 1) was determined to define the interpretative criteria for resistance, susceptibility and intermediate resistance and to compare with standard tests. Bacteria with resistance to ampicillin and carbenicillin as determined by standard tests gave $C_{i}$ values ranging from 0 to 10 , whereas susceptible isolates usually gave very high $C_{i}$ values $\left(>10^{3}\right)$, as dead cells were highly fluorescent with both dyes. The bottom limit of the $C_{i}$ value for susceptibility was arbitrarily established at 500 , as some susceptible isolates or samples had $\mathrm{C}_{\mathrm{i}}$ values just below 1000. Unfortunately, no bacteria were resistant to ceftriaxone, ceftazidime, gentamicin, or vancomycin; instead the interpretative criteria for ampicillin and carbenicillin were applied also to these four antibiotics. The ranges of $\mathrm{C}_{\mathrm{i}}$ values associated 
Table 1. Ranges of $\mathrm{C}_{\mathrm{i}}$ values determining the interpretative categories for susceptibility, intermediate resistance and resistance

\begin{tabular}{lccc}
\hline & \multicolumn{3}{c}{$\mathrm{C}_{\mathrm{i}}$ indicating } \\
\cline { 2 - 4 } Antibiotics & Susceptibility & $\begin{array}{c}\text { Intermediate } \\
\text { resistance }\end{array}$ & Resistance \\
\hline Ampicillin & $>500$ & $10-500$ & $0-10$ \\
Carbenicillin & $>500$ & $10-500$ & $0-10$ \\
Ceftriaxone & $>500$ & $10-500$ & $0-10$ \\
Ceftazidime & $>500$ & $10-500$ & $0-10$ \\
Gentamicin & $>500$ & $10-500$ & $0-10$ \\
Nitrofurantoin & $>10$ & - & $0-10$ \\
Norfloxacin & $>20$ & $10-20$ & $0-10$ \\
Oxacillin & $>500$ & $50-500$ & $0-50$ \\
Penicillin G & $>50$ & $50-100$ & $0-50$ \\
Tetracycline & $>50$ & $10-50$ & $0-10$ \\
Co-trimoxazole & $>500$ & $20-50$ & $0-20$ \\
Vancomycin & $10-500$ & $0-10$ \\
\hline
\end{tabular}

with resistance and susceptibility to nitrofurantoin, norfloxacin, oxacillin, penicillin $\mathrm{G}$, tetracycline and cotrimoxazole were different from those for ampicillin and carbenicillin. Resistance to nitrofurantoin, as determined by standard tests, was associated with $\mathrm{C}_{\mathrm{i}}$ values ranging from 0 to 10 , and susceptibility with values $>10$; resistance to tetracycline was associated with $C_{i}$ values of $0-10$ and susceptibility with values $>50$. The $\mathrm{C}_{\mathrm{i}}$ values for intermediate resistance were defined as being those associated with resistance but below those associated with susceptibility.

\section{FC-AST on control strains}

E. coli ATCC 25922. The light-scattering profile of untreated E. coli cultures (Fig. 1a) showed a homogeneous population of cells with intact membranes, with $>99 \%$ of these organisms impermeable to fluorescent dyes (Fig. 1b). After incubation for $2 \mathrm{~h}$ with ampicillin, the light-scattering profile became more dispersed (Fig. 1c), indicating important morphological changes; $>90 \%$ of the cells detected became permeable to the fluorescent dyes. Examination by epifluorescent microscopy revealed a high proportion of elongated and clustered cells (not shown). Bacteria treated with ceftazidime and ceftriaxone generated similar results (data not shown). These changes were easily detected and quantified by $\mathrm{FC}$, confirming several other reports $[2,5,9,10]$. Exposure to gentamicin did not significantly affect the light-scattering profile (Fig. 1e); nevertheless, susceptibility to this antibiotic could be shown, as $>80 \%$ of the cells detected by FC were fluorescent (Fig. 1f). Interestingly, two fluorescent populations appeared on the bi-parametric histogram, one was brighter with PI, whereas the other was brighter with $\operatorname{DiBAC}_{4}(3)$. With cotrimoxazole (trimethoprim:sulphamethoxazole at a 1:19 ratio), the light-scattering profile changed to generate a comet-tale pattern, reflecting the presence of enlarged cells (Fig. 1g). Although a significant number of fluorescent cells were detected in acquisition region II $(9.6 \%)$, a large percentage remained unstained after the $2 \mathrm{~h}$ of treatment (Fig. 1h).

Pseudomonas aeruginosa ATCC 27853. In contrast to E. coli, most untreated $P$. aeruginosa ATCC 27853 cells had an intrinsic permeability to the fluorescent dyes, with $43 \%$ of cells being detected in the acquisition region II designated for the dead cells (Fig. 2b). Similar results were observed with the two clinical
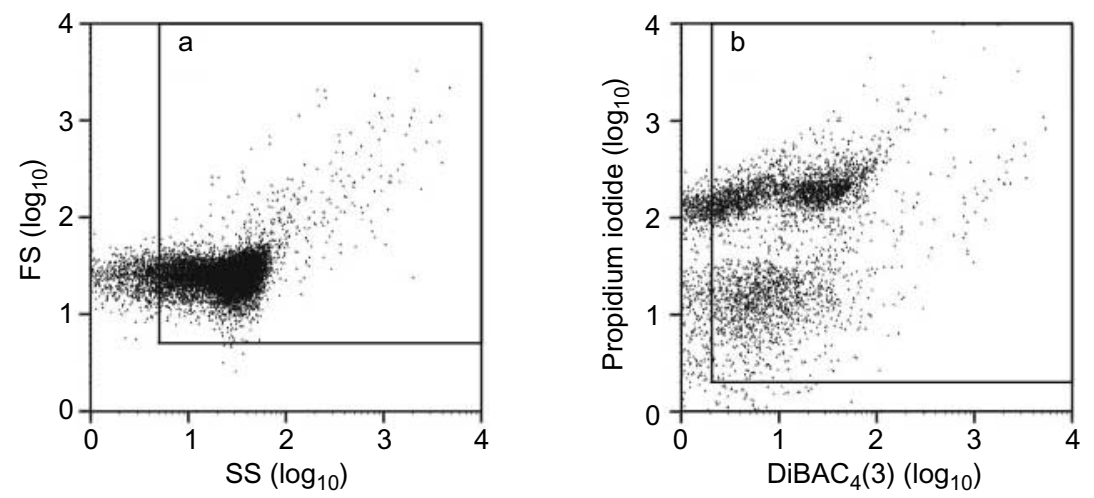

Fig. 2. Light-scattering profile (a) and bi-parametric histogram (b) $\left(\mathrm{DiBAC}_{4}(3)\right.$ versus $\left.\mathrm{PI}\right)$ for untreated P. aeruginosa ATCC 27853. No significant basal fluorescence was recorded (untreated and unstained control; data not shown). The proportion of dead cells (region II), recorded in panel b, was $43 \%$. 
P. aeruginosa strains with 15 and $70 \%$ of the cells in the untreated stained samples becoming fluorescent. No significant fluorescence was detected in the absence of fluorescent dyes, indicating that this behaviour could not be attributed to endogenous fluorescent molecules or organelles. Because of the high proportion of 'dead' cells in untreated samples, results with the treated samples were inconsistent and were excluded from analysis.

Gram-positive control bacteria. A high proportion $(>50 \%)$ of live log-phase gram-positive bacterial cells, including two $S$. aureus strains and two Ent. faecalis strains, were slightly permeable to PI (Fig. 3). However, these fluorescent events were not included in the acquisition region II. S. aureus ATCC 29213 is resistant to penicillin $\mathrm{G}$ and susceptible to the other five antibiotics

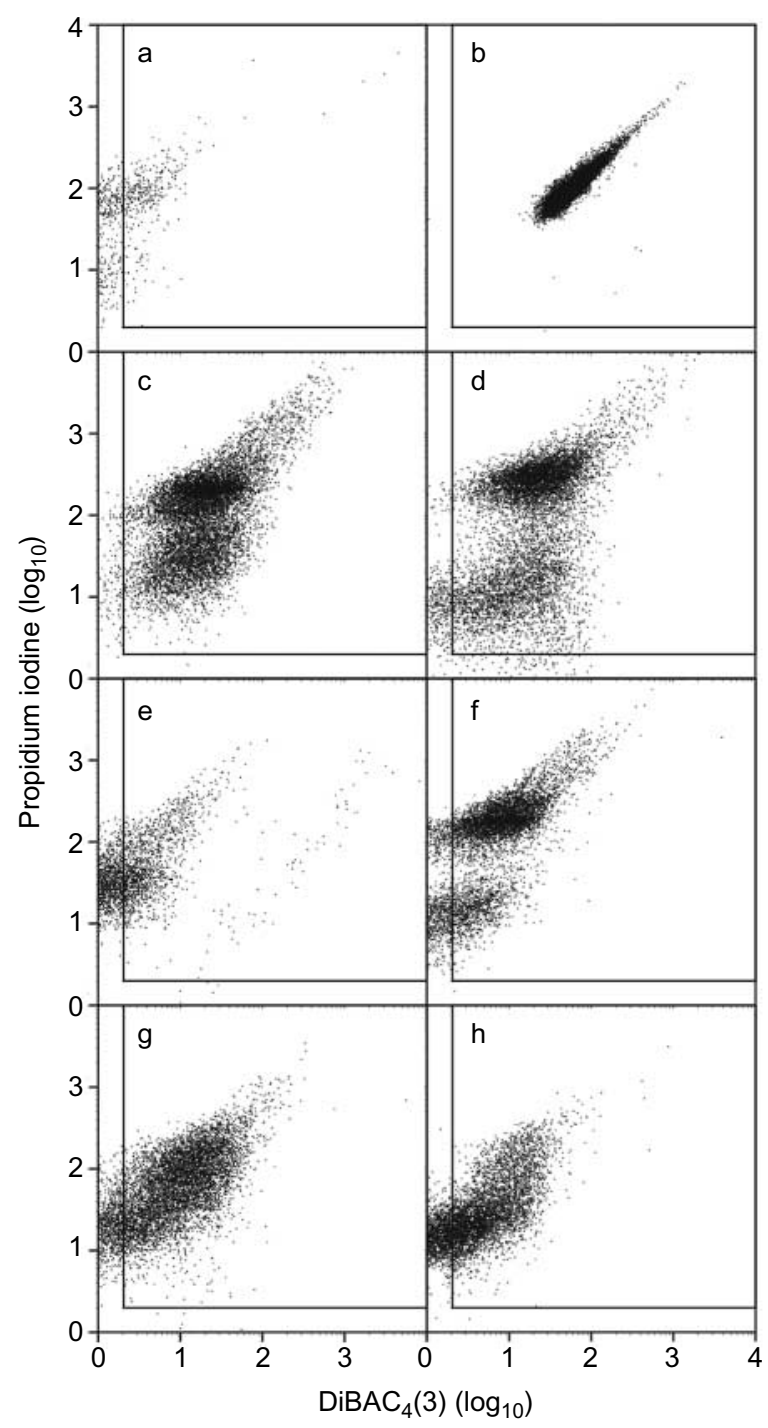

Fig. 3. Bi-parametric histograms $\left(\mathrm{DiBAC}_{4}(3)\right.$ versus $\left.\mathrm{PI}\right)$ for $S$. aureus ATCC 29213 untreated (a), permeabilised for $5 \mathrm{~min}$ with ethanol $70 \%(b)$, or treated for $2 \mathrm{~h}$ with vancomycin (c), oxacillin (d), penicillin $G(\mathbf{e})$, nitrofurantoin (f), norfloxacin (g) or co-trimoxazole (h), and analysed by FC. No significant basal fluorescence was recorded (data not shown). The proportions of dead cells recorded (region II) in panels $\mathbf{a}-\mathbf{h}$ were $3.1 \%, 99 \%, 97 \%, 84 \%, 14 \%, 56 \%, 61 \%$ and $43 \%$, respectively. tested as determined by standard ASTs. As illustrated in Fig. 3, the FC-AST correctly identified this pattern.

\section{FC-AST on UTI specimens}

Several clinical bacterial specimens were collected from UTIs. Although E. coli accounts for $>80 \%$ of UTIs, this study aimed to gather representatives of many different types of bacterial UTIs. Fig. 4 shows the susceptibility of E. coli 15462, a urine isolate, to ceftriaxone, gentamicin, norfloxacin and nitrofurantoin, and its resistance to ampicillin and co-trimoxazole, as determined by FC-AST. Both conventional susceptibility test methods supported these results.

To bypass the overnight incubation period to obtain single colonies, FC-AST was applied directly to 19 urine samples infected with $>10^{5} \mathrm{cfu} / \mathrm{ml}$, and representing four different species. With urine samples infected with E. coli, the FC-AST correlated at $98 \%$ (13 specimens, 114 tests) and gave only two minor errors. With urine samples infected with organisms other than E. coli (six specimens, 48 tests), five

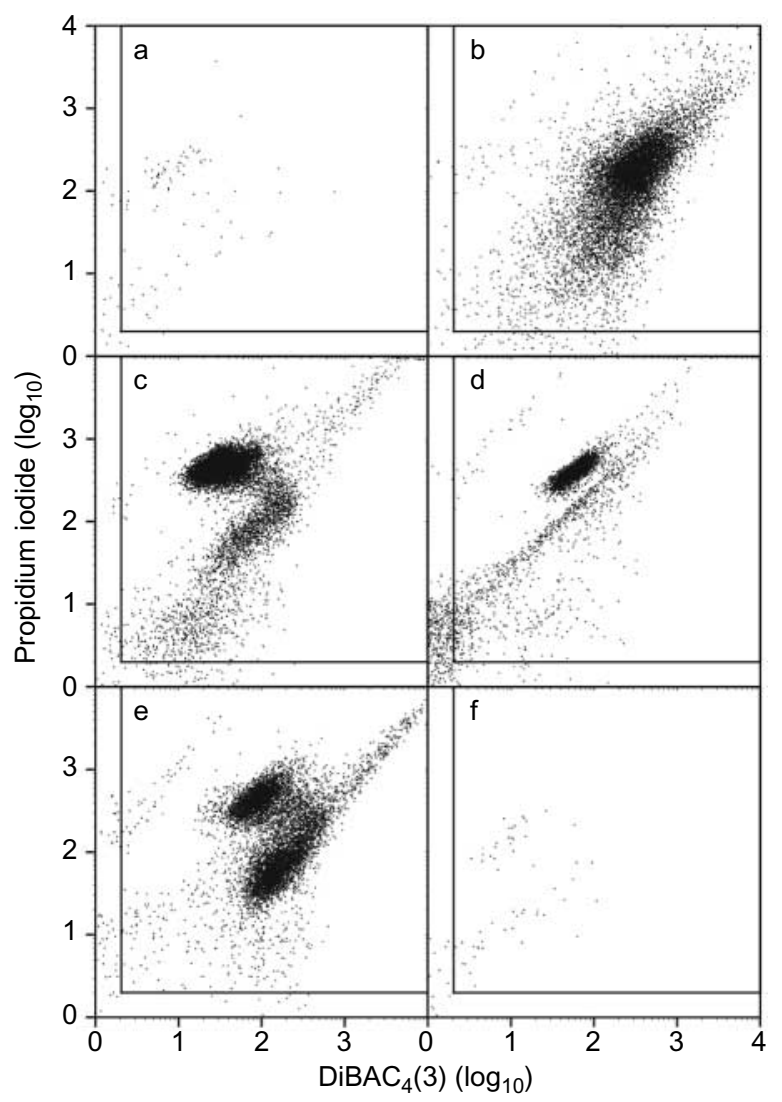

Fig. 4. Bi-parametric histograms $\left(\mathrm{DiBAC}_{4}(3)\right.$ versus $\left.\mathrm{PI}\right)$ for the urine isolate $E$. coli 15462 treated for $2 \mathrm{~h}$ with ampicillin (a), ceftriaxone (b), gentamicin (c), norfloxacin (d), nitrofurantoin (e) or co-trimoxazole (f), and analysed by FC. The proportion of non-fluorescent cells in the untreated sample was $99.1 \%$, whereas $99.8 \%$ of the cells in the ethanol-treated sample were fluorescent with both dyes (data not shown). The proportions of dead cells recorded (region II) in panels a-f were $0.8 \%, 94 \%, 94 \%, 88 \%, 70 \%$ and $0.7 \%$, respectively. 
Table 2. FC-AST on control strains, urine isolates and urine samples

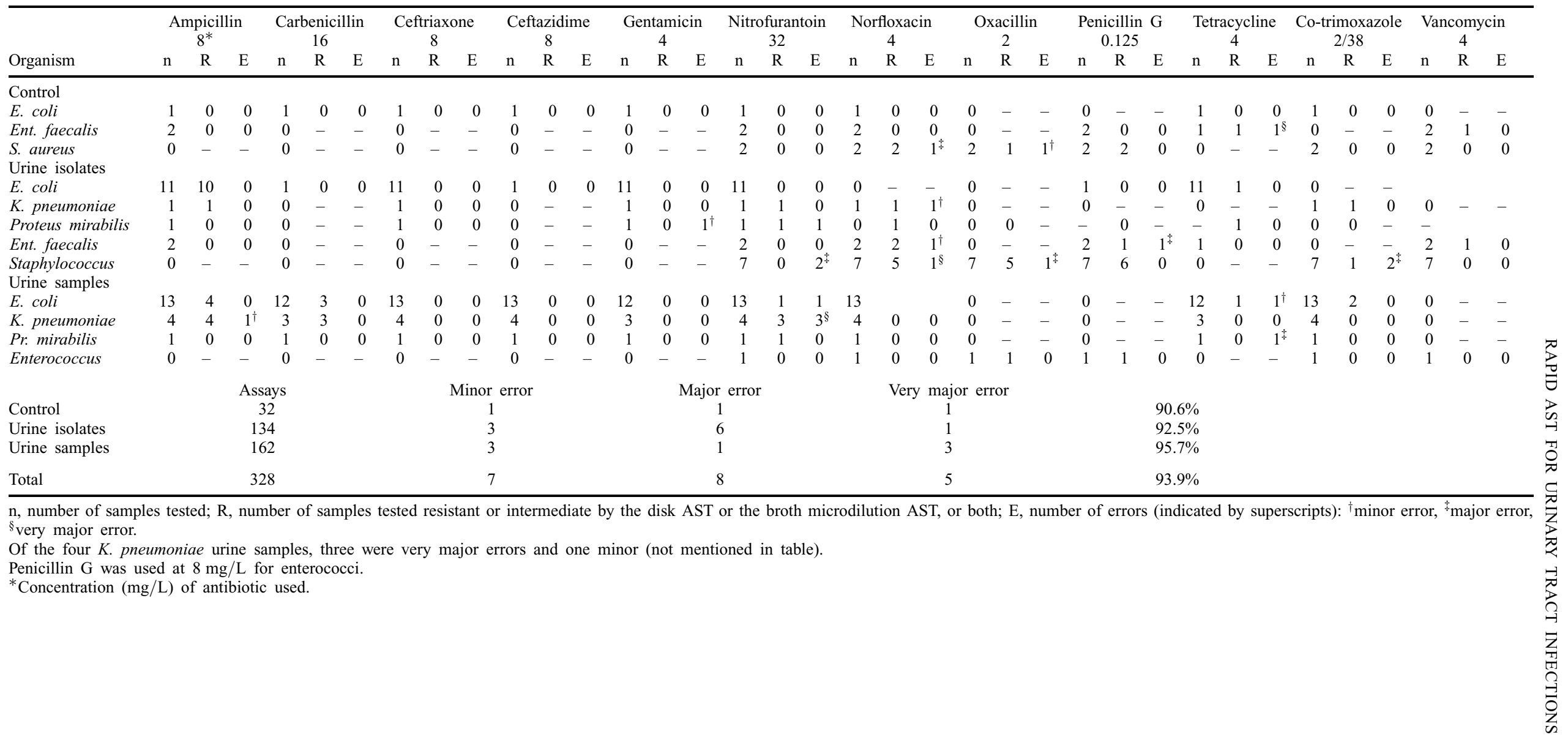




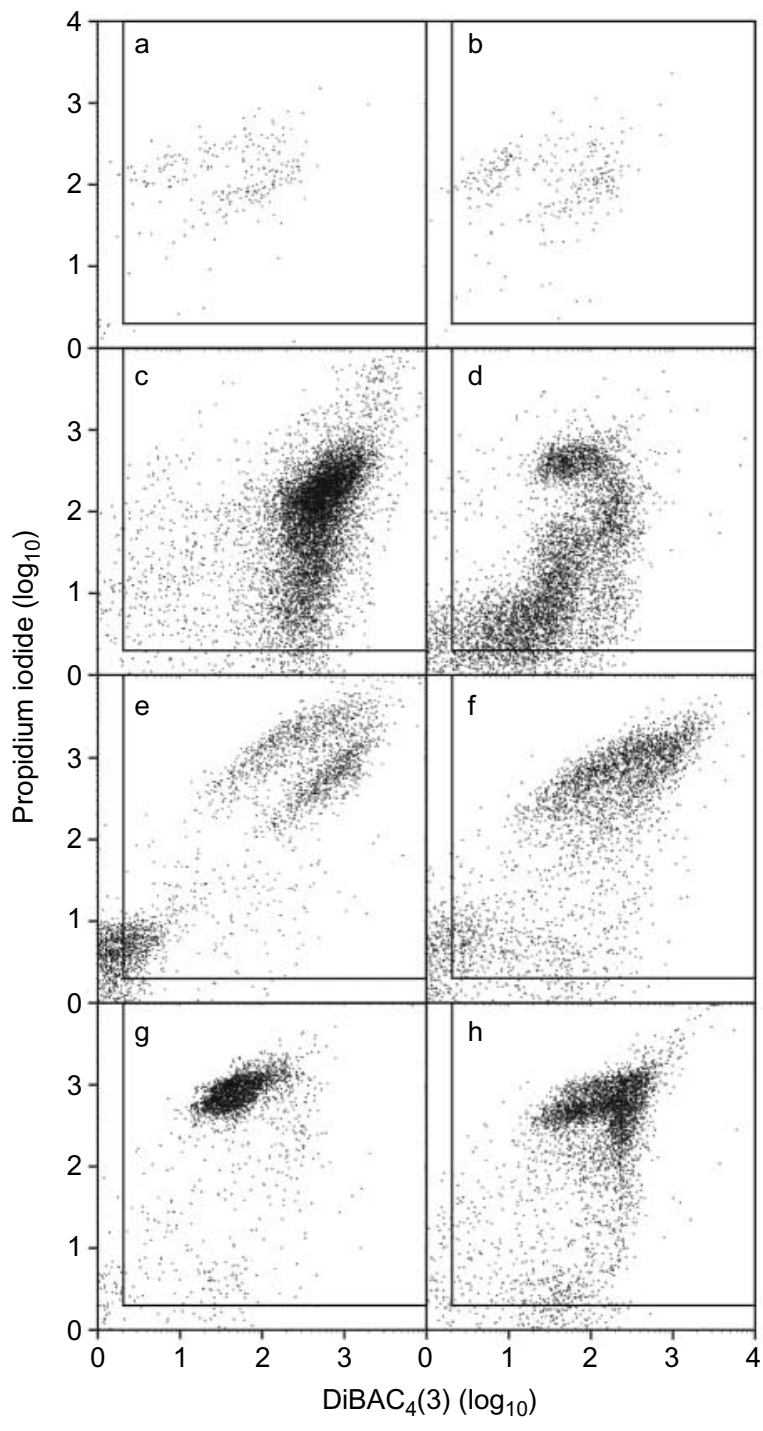

Fig. 5. Bi-parametric histograms $\left(\operatorname{DiBAC}_{4}(3)\right.$ versus $\left.\mathrm{PI}\right)$ for a urine sample, identified later as containing $K$. pneumoniae 43450 , treated for $2 \mathrm{~h}$ with ampicillin (a), carbenicillin (b), ceftriaxone (c), gentamicin (d), norfloxacin (e), nitrofurantoin (f), tetracycline (g) or co-trimoxazole (h), and analysed by FC. The proportion of non-fluorescent cells in the untreated sample was $94.4 \%$, whereas $99.6 \%$ of the cells in the ethanol-treated sample were fluorescent with both dyes (data not shown). The proportions of dead cells recorded (region II) in panels $\mathbf{a}-\mathbf{h}$ were $2.2 \%, 2.8 \%, 85 \%, 54 \%, 30 \%, 22 \%, 28 \%$ and $43 \%$, respectively.

discrepancies were observed with the conventional assays (90\% agreement) (Table 2). Three of these were very major errors and involved nitrofurantoin and Klebsiella pneumoniae. Fig. 5 shows FC-AST results on one of these samples. Susceptibility was observed for this strain to ceftriaxone, ceftazidime (not shown), gentamicin, norfloxacin, nitrofurantoin, tetracycline and co-trimoxazole, and resistance to ampicillin and carbenicillin with both the FC-AST and standard tests, whilst disagreement was seen only for nitrofurantoin (Fig. 5f).

Incubation of $100 \mu \mathrm{l}$ of urine (containing $>10^{5} \mathrm{cfu} / \mathrm{ml}$ from UTI patients) in $5 \mathrm{ml}$ of MHSC consistently yielded visible growth after $90-120 \mathrm{~min}$ at $37^{\circ} \mathrm{C}$, whereas no visible growth was observed with urine from a healthy individual. The number of events recorded by $\mathrm{FC}$ of these urine samples was equivalent to that of filtered PBS (data not shown).

\section{Discussion}

The capacity of the fluorescent probes to detect antibiotic effects on individual cells, and the use of flow cytometry to measure and enumerate these effects rapidly was a major factor in establishing the FC-AST protocol. Other reports also indicated the superiority of PI and $\mathrm{DiBAC}_{4}(3)$ over other probes to determine bacterial mortality $[11,12]$. Although not all antibiotics target the membrane, their effects such as the inhibition of protein synthesis - can have rapid but indirect effects on this structure [2]. The use of two mortality dyes increases the confidence that cells harbouring double fluorescence are nonviable. Thus, Nebe-von Caron et al. [13] showed that some bacteria stained only by $\operatorname{DiBAC}_{4}(3)$ may have lost membrane potential but not membrane integrity, and that a significant proportion of these could be recovered on solid media. However, cells stained with both $\mathrm{PI}$ and $\mathrm{DiBAC}_{4}(3)$ showed no recovery. The very short incubation time $(5-10 \mathrm{~min})$ required for staining, and the fact that no washing, permeabilising or fixation steps are necessary before FC analysis are also strong advantages for the use of these two fluorochromes.

The incubation time with antibiotics is an important factor to consider in the development of a rapid FCAST. Although morphological changes can be observed by FC within $30 \mathrm{~min}$ with $\beta$-lactam agents, the present study found that $2 \mathrm{~h}$ at $37^{\circ} \mathrm{C}$ was the minimum period required to allow reliable results with all the bacteriaantibiotic combinations tested. This short exposure was sufficient to produce a significant decrease of bacterial concentrations as determined by plate counting, from $10^{8} \mathrm{cfu} / \mathrm{ml}$ to $10^{3}-10^{6} \mathrm{cfu} / \mathrm{ml}$, depending on the type of antibiotic $\left(10^{3} \mathrm{cfu} / \mathrm{ml}\right.$ was the detection limit) (not shown).

The interpretation criteria for susceptibility and resistance are well defined with the two conventional methods, but interpretative criteria for the FC-AST had to be established. While susceptibility could be observed qualitatively with some antibiotics (e.g., $\beta$ lactams) in the form of significant changes in the lightscattering profiles, a quantitative measurement was sought to give a more objective determination. This measure was based on the number of cells fluorescent for both dyes and the mean fluorescence intensity (MFI) of events measured in acquisition region I, and was compared between antibiotic-treated and -nontreated samples giving the ratio $C_{i}$. The ranges of the $\mathrm{C}_{\mathrm{i}}$ values that defined resistance, susceptibility and 
intermediate resistance for FC-AST were established by comparison with standard tests. However, some of the $\mathrm{C}_{\mathrm{i}}$ range were not defined, because resistant isolates were not available (e.g., with gentamicin, vancomycin, ceftriaxone, ceftazidime). $\mathrm{C}_{\mathrm{i}}$ values between 0 and 10 correlated well with resistance and $C_{i}$ values $>1000$ correlated with susceptibility. Nine $(45 \%)$ of the 20 discrepancies were caused by $\mathrm{C}_{\mathrm{i}}$ values that were between 10 and 1000 .

FC-AST was first evaluated with six ATCC control strains representing four bacterial species frequently encountered in UTIs: E. coli, P. aeruginosa, Ent. faecalis and $S$. aureus. The method was not suitable for $P$. aeruginosa, where live cells stained with both dyes, especially PI. P. aeruginosa is a strict aerobe and it rapidly de-energises when deprived of an energy source, resulting in enhanced permeability of its outer membrane (R. E. W. Hancock, Department of Microbiology and Immunology, University of British Columbia, Vancouver, Canada, personal communication). This bacterium is also sensitive to temperature changes such as arise when standing without agitation at room temperature or when incubated on ice [14]. Such conditions occurred during the FC-AST. Therefore, modifications of the protocol might be tested, such as bypassing the PBS washing step, by adding the fluorescent probes directly in the cultures at the end of the antibiotic treatment, and by staining for $\leqslant 5 \mathrm{~min}$ at $37^{\circ} \mathrm{C}$.

The changes in light-scattering and fluorescence profiles were specific for each antibiotic or class. The four $\beta$-lactam antibiotics - ampicillin, carbenicillin, ceftriaxone and ceftazidime that were used mainly in this study against gram-negative bacteria - produced similar changes to one another in the light-scattering profile, usually with $>80 \%$ of the cells becoming fluorescent with one or both dyes. Two other $\beta$-lactam antibiotics, penicillin $\mathrm{G}$ and oxacillin, are used against gram-positive bacteria, and showed a different pattern: untreated logarithmic phase cells displayed a significant (but weak) PI fluorescence, whereas treated, susceptible cells showed a bright staining with $\operatorname{DiBAC}_{4}(3)$, thus underscoring the advantage of using a combination of mortality dyes. Although the light-scattering profile was not affected by gentamicin, a large percentage of cells became fluorescent with both dyes. In contrast to the behaviour with $\beta$-lactam agents, gentamicin generated two populations as resolved by fluorescence profiles. This probably reflects the mechanism of action of gentamicin, which first causes the loss of membrane potential, then affects membrane integrity.

Taking the FC-AST results for the five control strains together with those for urine samples and isolates, there were 20 discrepancies among 328 individual comparisons $(93.9 \%$ agreement). Non-E. coli strains accounted for 18 of those discrepancies, and $13(65 \%)$ of these 20 occurred when norfloxacin, nitrofurantoin and tetracy- cline were tested. Low percentages of fluorescent cells (5-50\%) were generally obtained from susceptible strains incubated with nitrofurantoin and tetracycline, which are bacteriostatic, and this could have resulted in a misinterpretation of susceptibility.

The goal of this study was to evaluate the feasibility of a FC-AST in a clinical setting, not an exhaustive statistical comparison with standard antimicrobial tests. Therefore the aim was to gather many different bacterial species involved in clinical urine infections, especially those with wide resistance. The results showed that FC-AST has similar reliability when testing either urine isolates (10 discrepancies in 134 tests, $93 \%$ or urine samples (7 discrepancies in 162 tests; 96\%). FC-AST applied directly to urine samples instead of isolates would accelerate antimicrobial susceptibility testing, as it does not require the overnight incubation for isolating colonies on solid media [15-17].

In our hands, the protocol required $5 \mathrm{~h}$ for the analysis of up to 20 samples (as cytometers in current use acquire data in a serial fashion), instead of the $24-48 \mathrm{~h}$ for standard tests. Therefore, FC-AST could be a rapid and accurate alternative to conventional methods, and could benefit health-care systems by accelerating urine analysis.

We thank Dr Michel Laverdière and Christiane Restieri, from the Department of Infectious Diseases and Microbiology, Hôpital Maisonneuve-Rosemont (University of Montreal). Ceftriaxone was a gift from Hoffman-La Roche. Funding for this research was provided by the National Science and Engineering Research Council of Canada.

\section{References}

1. McFeters GA, Yu FP, Pyle BH, Stewart PS. Physiological assessment of bacteria using fluorochromes. $J$ Microbiol Methods 1995; 21: 1-13.

2. Gant VA, Warnes G, Phillips I, Savidge GF. The application of flow cytometry to the study of bacterial responses to antibiotics. J Med Microbiol 1993; 39: 147-154.

3. Langsrud S, Sundheim G. Flow cytometry for rapid assessment of viability after exposure to a quaternary ammonium compound. J Appl Bacteriol 1996; 81: 411-418.

4. Deere D, Porter J, Edwards C, Pickup R. Evaluation of the suitability of bis-(1,3-dibutylbarbituric acid) trimethine oxonol, (DiBAC $\left._{4}(3)-\right)$, for the flow cytometric assessment of bacterial viability. FEMS Microbiol Lett 1995; 130: 165-170.

5. Jepras RI, Paul FE, Pearson SC, Wilkinson MJ. Rapid assessment of antibiotic effects on Escherichia coli by bis(1,3-dibutylbarbituric acid) trimethine oxonol and flow cytometry. Antimicrob Agents Chemother 1997; 41: 2001-2005.

6. Davey HM, Kell DB. Flow cytometry and cell sorting of heterogeneous microbial populations: the importance of single cell analyses. Microbiol Rev 1996; 60: 641-696.

7. Shapiro HM. Practical flow cytometry, 2nd edn. New York, Alan R. Liss. 1988.

8. National Committee for Clinical Laboratory Standards. Performance standards for antimicrobial disk susceptibility tests M2-A6. Methods for dilution antimicrobial susceptibility tests for bacteria that grow aerobically M7-A4. Ninth informational supplement M100-S9. Villanova, PA, NCCLS. 1999.

9. Walberg M, Gaustad P, Steen HB. Rapid flow cytometric assessment of mecillinam and ampicillin bacterial susceptibility. J Antimicrob Chemother 1996; 37: 1063-1075. 
10. Walberg M, Gaustad P, Steen HB. Rapid assessment of ceftazidime, ciprofloxacin, and gentamicin susceptibility in exponentially-growing $E$. coli cells by means of flow cytometry. Cytometry 1997; 27: 169-178.

11. Comas J, Vives-Rego J. Enumeration, viability and heterogeneity in Staphylococcus aureus cultures by flow cytometry. J Microbiol Methods 1998; 32: 45-53.

12. Mason DJ, Power EGM, Talsania H, Phillips I, Gant VA. Antibacterial action of ciprofloxacin. Antimicrob Agents Chemother 1995; 39: 2752-2758.

13. Nebe-Von Caron G, Stephens P, Badley RA. Assessment of bacterial viability status by flow cytometry and single cell sorting. J Appl Microbiol 1998; 84: 988-998.

14. Lei Y, Satake S, Ishii J, Nakae T. Factors that influence the permeability assay of the outer membrane of Pseudomonas aeruginosa. FEMS Microbiol Lett 1991; 80: 337-340.

15. Gillenwater JY, Clark MM. Tentative direct antimicrobial susceptibility testing in urine. J Urology 1996; 156: 149-153.

16. Johnson JR, Tiu FS, Stamm WE. Direct antimicrobial susceptibility testing for acute urinary tract infections in women. J Clin Microbiol 1995; 33: 2316-2323.

17. Oakes AR, Badger R, Grove DI. Comparison of direct and standardised testing of infected urine for antimicrobial susceptibilities by disk diffusion. J Clin Microbiol 1994; 32: 40-45. 